\title{
BMJ Global Health The mixed effects of a package of multilevel interventions on the health and care of pregnant women in Zinder, Niger
}

Cesaire T Ouedraogo (D) , ${ }^{1,2} \mathrm{~K}$ Ryan Wessells, ${ }^{1}$ Rebecca R Young, ${ }^{1}$ Ibrahim Foungotin Bamba, ${ }^{2}$ M Thierno Faye, ${ }^{2}$ Ndiaye Banda, ${ }^{3}$ Sonja Y Hess $^{1}$

To cite: Ouedraogo CT, Wessells KR, Young RR, et al. The mixed effects of a package of multilevel interventions on the health and care of pregnant women in Zinder, Niger. BMJ Global Health 2019;4:e001200. doi:10.1136/ bmjgh-2018-001200

Handling editor Soumitra S Bhuyan

- Additional material is published online only. To view please visit the journal online (http://dx.doi.org/10.1136/ bmjgh-2018-001200).

Received 4 October 2018 Revised 8 June 2019 Accepted 10 June 2019

\section{Check for updates}

(c) Author(s) (or their employer(s)) 2019. Re-use permitted under CC BY-NC. No commercial re-use. See rights and permissions. Published by BMJ.

${ }^{1}$ Institute for Global Nutrition, Department of Nutrition, University of California Davis, Davis, California, USA

${ }^{2}$ Helen Keller International, Niamey, Niger

${ }^{3}$ Nutrition International, Dakar, Senegal

Correspondence to

Dr Sonja Y Hess;

syhess@ucdavis.edu

\section{ABSTRACT}

Background Anaemia is prevalent among pregnant women in rural Niger and antenatal care (ANC) attendance is suboptimal. We designed a programmatic intervention including community-based behaviour change communication, provision of essential drugs (including iron folic acid (IFA) supplements) and quality improvement activities at selected integrated health centres (IHCs). Objective To assess the impact of the programmatic intervention on: (1) utilisation of ANC, (2) adherence to daily IFA supplementation and (3) prevalence of adequate gestational weight gain (GWG) and anaemia among pregnant women in Zinder, Niger.

Methods Using a quasi-experimental study design comparing a cohort of women at baseline to another cohort of women at endline, $18 \mathrm{IHCs}$ and surrounding villages were randomly assigned to time of enrolment over 1 year. A baseline survey was implemented among randomly selected pregnant women in 68 village clusters. Subsequently, the intervention was rolled out and an endline survey was implemented 6 months later in the same villages.

Results Mean age in the baseline $(n=1385)$ and endline $(n=922)$ surveys was $25.8 \pm 6.4$ years. The percentage of pregnant women who reported attending any number of ANC and an adequate number of ANC for their gestational age, respectively, was not significantly different between the endline and the baseline surveys. Pregnant women in the endline survey were more likely to have received IFA $(60.0 \%$ vs $45.8 \%, 0 \mathrm{R}: 2.7(1.2,6.1))$; and the proportion of pregnant women who reportedly consumed IFA daily in the previous 7 days was significantly higher in the endline than in the baseline survey $(46.4 \%$ vs $32.8 \%$, OR: $2.8(1.2,6.5))$. There was no impact on the prevalence of adequate GWG or anaemia.

Conclusions The programmatic intervention resulted in a modest increase in the number of pregnant women who reported receiving and consuming IFA supplements as recommended, but did not affect ANC attendance and nutritional status.

\section{INTRODUCTION}

Optimal healthcare during pregnancy is important for the well-being of the mother

\section{Key questions}

What is already known?

- Interventions to improve antenatal care (ANC) services and the health of pregnant women require a multilevel intervention strategy.

What are the new findings?

- There was a high prevalence of anaemia and inadequate gestational weight gain (GWG) among pregnant women in rural Zinder, Niger.

- After 6 months of a package of multilevel interventions including bimonthly community-based behaviour change communication activities in each selected village, provision of essential drugs (including iron folic acid (IFA)) and supplies, and quality improvement activities at selected integrated health centres (IHCs), there was a modest increase in the proportion of pregnant women who reported receiving and consuming IFA supplements as recommended (ie, consumed IFA supplement daily in the previous 7 days).

- No impact was found on overall ANC seeking, GWG mid-upper arm circumference and haemoglobin concentration.

What do the new findings imply?

- The anaemia status and GWG is inadequate among many pregnant women in rural Zinder and further research is needed in the type of interventions that could address these public health issues.

and the development of the fetus. ${ }^{1}$ Antenatal care (ANC) represents an opportunity for pregnant women to receive a broad range of health promotion and prevention services and any required medical treatment. ${ }^{2}$ Until 2016, the WHO recommended at least four ANC visits for uncomplicated pregnancies, with the first visit occurring during the first trimester of pregnancy. ${ }^{3}$ In the most recent WHO recommendations on ANC, the recommended number of contacts was increased to eight, with the initial contact in the first 
trimester, two contacts in the second trimester and five in the third trimester. ${ }^{4}$ According to available scientific evidence, the increased number of contacts may facilitate comprehensive assessment of the pregnant woman's health status and provide an opportunity to intervene early if pregnancy-related conditions are identified. ${ }^{4}$ However, in Niger, only 33\% of women in the 2012 National Demographic and Health Survey reported having attended at least four ANC visits during their last pregnancy. ${ }^{5}$

Previous studies that have examined the factors affecting ANC attendance indicated that barriers to ANC attendance include accessibility of ANC (eg, transportation, clinic fees), beliefs and attitudes towards ANC (eg, receiving a quantity of medicine that takes a very long time to finish, limited understanding of the services during ANC visits), poor quality of ANC services (eg, long waiting time, lack of medical equipment, poor health staff attitude including insensitivity, disrespect and abuse) and personal factors (eg, familial support, relationship with healthcare providers, fear of public disclosure of the pregnancy especially for young women).$^{6-8}$ However, a systematic review on various types of health system and community level interventions for improving ANC coverage and health outcomes has shown that combining community-based interventions and health systems-related interventions compared with no intervention led to increases in ANC coverage of at least one visit. ${ }^{9}$

Although the impact of some antenatal interventions to reduce maternal and infant mortality still remain unclear, ANC has the potential, at least in theory, to reduce maternal morbidity and mortality directly through the detection and treatment of pregnancy-related or intercurrent illness, or indirectly through the detection of women at increased risk of complications of delivery and ensuring that they deliver in a suitably equipped facility. ${ }^{10}$ The potential of ANC to reduce maternal and infant mortality is presumably greater in low-income countries where morbidity and mortality are higher. In Niger, the total fertility rate of 7.4 is one of the highest in the world. ${ }^{11}$ The maternal mortality rate (520 deaths per 100000 live births) and the infant mortality rate (51 deaths per 1000 live births between birth and 1 year of age) are also among the highest in the world. ${ }^{12}$

Often delivered through ANC, iron folic acid (IFA) supplementation is recommended as an important antenatal intervention to improve iron and folate status and reduce the risk of anaemia. IFA supplementation is associated with a reduced risk of preterm birth, low birth weight, neonatal death and congenital anomalies. ${ }^{13} 14$ The WHO currently recommend daily IFA supplementation (30-60 mg elemental iron and $400 \mu \mathrm{g}$ folic acid) during pregnancy starting as early as possible to prevent maternal anaemia, puerperal sepsis, low birth weight, preterm birth, maternal anaemia and iron deficiency. ${ }^{15}$ Despite these recommendations, in the 2012 Demographic and Health Survey $58.6 \%$ of pregnant women were anaemic in Niger, and only $29 \%$ of women reported consuming IFA supplements for $\geq 90$ days during their most recent pregnancy. ${ }^{5}$

The present study, entitled the Niger Maternal Nutrition $(\mathrm{NiMaNu})$ project, was implemented in the Zinder region of Niger. The NiMaNu project was a programmatic intervention to improve ANC services, with a research component to assess and evaluate the impacts of the intervention. To initiate the project, formative research was conducted to: (1) understand knowledge, attitudes and practices (KAP) among pregnant women related to ANC, IFA supplementation and health during pregnancy and (2) assess the quality of currently provided ANC services. The findings of the previously reported formative research were used to guide the development and implementation of a programmatic intervention designed to increase the proportion of pregnant women who receive ANC services and to increase IFA supplementation of pregnant women in rural Zinder, ${ }^{16}$ which then was piloted in two health districts of the Zinder region in Niger, as described in more detail below.

The objective of the present study was to assess the impacts of the programmatic intervention. In particular, we aimed to assess: (1) the utilisation of ANC services, (2) the adherence to IFA supplementation as recommended, (3) women's KAP related to ANC and IFA before and after the programmatic intervention and (4) the effects of these changes on the prevalence of adequate gestational weight gain $(\mathrm{GWG})$, adequate mid-upper arm circumference (MUAC) and anaemia among pregnant women.

\section{METHODS}

\section{Study design, setting and population}

The NiMaNu project was approved by the National Ethics Committee in Niamey, Niger and the Institutional Review Board of the University of California, Davis, USA. In the presence of a neutral witness, consent materials were presented by multiple enumerators in both written and oral formats. A standardised informed consent procedure was used and documented with a written signature or a fingerprint. Participants received a locally appropriate gift such as a bar of soap to compensate for their time and effort. The trial was registered at www.clinicaltrials.gov as NCT01832688.

The NiMaNu project was designed as a quasiexperimental descriptive study comparing preintervention and postintervention cohorts of pregnant women. A multistage clustered sampling design was used for the baseline and endline surveys. Two health districts (Mirriah and Zinder) in the Zinder region were selected in collaboration with regional and health district personnel for convenience based on their accessibility, distance to Zinder, and the limited number and scope of interventions being implemented in the catchment area of the integrated health centres (IHCs) within these districts to avoid overlapping interventions conducted in the same area. In Niger, the health system in a district is 
organised at three levels: (1) health posts provide very basic outpatient curative and preventive care and are staffed primarily by community health workers, (2) IHCs serve several villages, provide outpatient and inpatient care, including labour and delivery, and are staffed by nurses, midwifes and sometimes physicians, and (3) a district hospital, provides diagnostics services, outpatient and inpatient curative care, and Caesarean sections, and is the level of referral for IHCs. ${ }^{17}{ }^{18}$ In the health district of Zinder, all eight IHCs serving rural communities were selected, and the remaining two IHCs were excluded because they only served urban areas. In Mirriah, 10 out of 36 IHCs were selected based on convenience sampling.

A statistician at the University of California, Davis stratified individual IHCs in groups of three by selected indicators (total population; district; distance to Zinder; number of health posts), and then randomly assigned individual IHCs to the order of enrolment. Within each IHC, 10 villages were randomly selected after stratification by distance from IHC and population size. First, the village where the IHC was located was automatically chosen for participation and one village containing a health post was randomly selected from all those with a health post that were within the catchment area of the IHC. Second, among the remaining villages in the catchment area of the health centre, four villages $\leq 10 \mathrm{~km}$ and four villages $>10 \mathrm{~km}$ from the IHC were randomly selected and randomised to the order of enrolment.
To assess the impacts of the programmatic intervention, a rolling baseline survey was implemented in 12 IHCs over the course of 1 year among randomly selected pregnant women in participating villages. Once the baseline survey was completed in the catchment area of an IHC, the programmatic intervention was implemented. Subsequently, the endline survey was implemented in each village 6 months after the completion of the village's baseline survey. The baseline survey was extended in parallel with the endline survey for 6 months to serve as a preand-post and time-matched comparison and to account for the impact of season (figure 1); thus the remaining 6 IHCs participated in the baseline survey only.

All the resident pregnant women in the selected villages (ie, women who lived in the study area for the 6 months prior to enrolment) and had no plans to move out of the study area within the next 2 months, were eligible to participate in the surveys and measurements, after providing written informed consent. Pregnant women who had severe illnesses warranting immediate hospital referral and those who were unable to provide consent due to impaired decision making ability were excluded.

The target enrolment was 77 women per IHC, to reach the sample size described in more detail below. Pregnant women were identified using a random walk method ${ }^{19}$ until 16-20 women per village were enrolled or until there were no more eligible pregnant women in the village. Randomly selected villages were visited by order

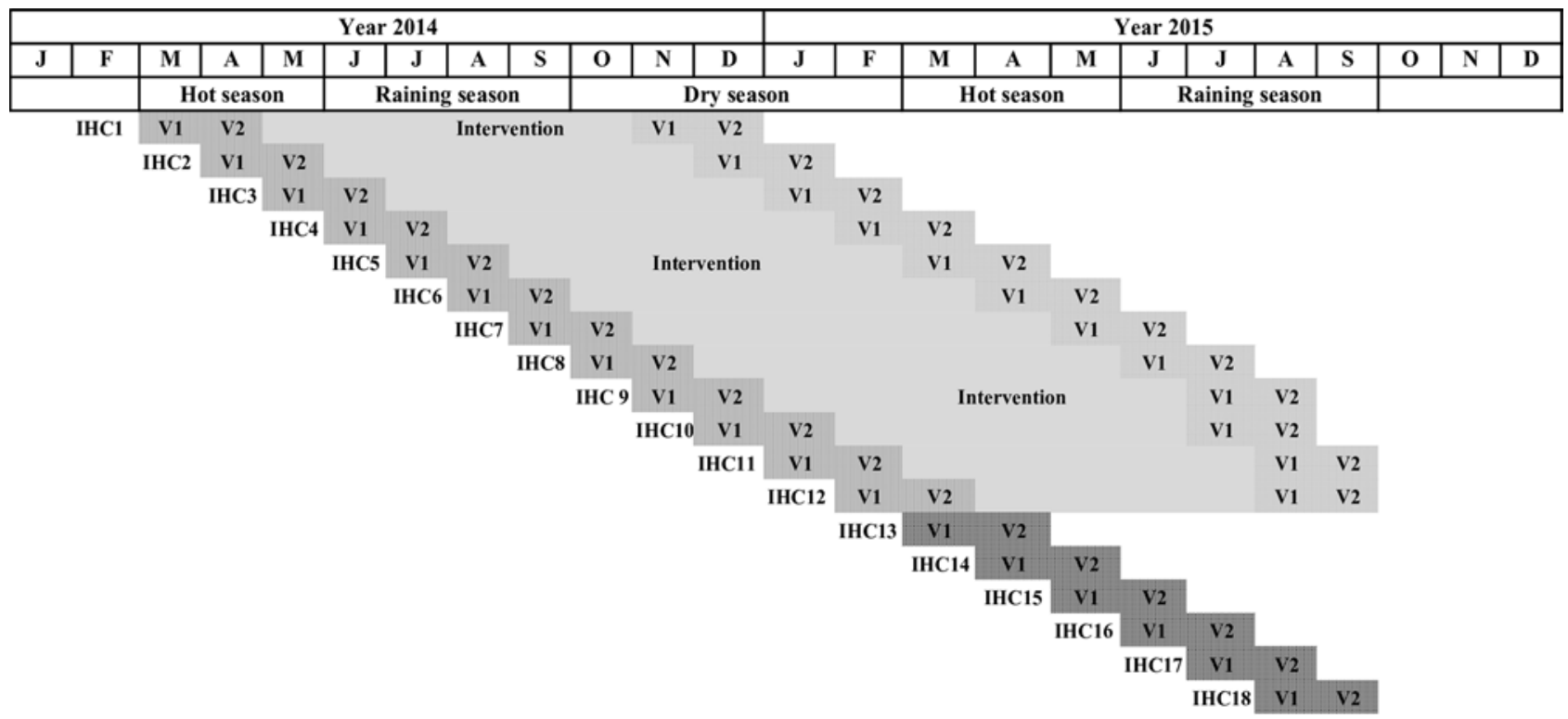

Baseline survey in intervention IHC

Baseline survey in control IHC

Endline survey in intervention IHC

Intervention Behavior change communication sessions in villages; quality improvement activities in the IHC and provision of adequate supplies and drugs to the IHC

V1 Visit 1

V2 Visit 2

Figure 1 Timeline of the baseline and endline surveys. 
of randomisation until the target sample size per IHC was reached. Participants in the baseline and endline surveys were not the same. A local guide accompanied the survey teams to help identify pregnant women during the random walk process. The random walk method was conducted independently for each survey.

\section{Baseline and endline surveys}

The baseline and the endline surveys started in March 2014 and November 2014, respectively, and both continued until September 2015. Each participant was contacted twice during the baseline phase to enable the determination of GWG. During the first contact, the participants were visited at their homes, where written consent was obtained. Information on last menstrual period, parity, maternal age, marital status, education and other indicators of socioeconomic status was collected. KAP relating to diet, health, pregnancy and ANC were assessed using a structured interview. Height, weight, MUAC and fundal height were measured using anthropometric equipment calibrated daily and by multiple enumerators who participated in regular standardisation sessions. Lightly clothed pregnant women were weighed to $50 \mathrm{~g}$ precision (SECA 874) in duplicate. Women's height (SECA 213, Seca, Hamburg, Germany), MUAC (ShorrTape Measuring Tape, Weigh and Measure, Olney, Maryland, USA) and symphysis-fundal height (SFH; ShorrTape Measuring Tape, Weigh and Measure, Olney, Maryland, USA) were measured in duplicate to $0.1 \mathrm{~cm}$ precision. If the two measurements were $>0.2 \mathrm{~kg}$ (weight) or $>0.5 \mathrm{~cm}$ apart (height, MUAC and SFH), a third measurement was taken and the mean of the two closest measurements was calculated.

Each participating pregnant woman was invited to a gathering space such as an IHC or school, or the houses of a village chief for a follow-up assessment $\sim 1$ month later, where anthropometric measurements and the KAP interviews were repeated and weight gain over the elapsed period was calculated. Capillary blood samples were collected for on-site assessment of haemoglobin $(\mathrm{Hb})$ concentration by HemoCue Hb 201+ (Hemocue, Lake Forest, California, USA). The survey questionnaires were developed in French and revised after pretesting. Interviews were conducted in Hausa (the predominant local language) or French.

Gestational age was estimated as a weighted average of the following obtained information: reported last menstrual period (by estimated number of months, lunar cycles and/or proximity to a religious or cultural event), time elapsed since pregnant woman started to feel or perceive fetal movements, and two fundal height measurements taken $\sim 1$ month apart. ${ }^{20} 21$ Trimester of pregnancy was defined as follows: first trimester: $<13$ weeks, second trimester: $\geq 13$ weeks to $<27$ weeks and third trimester: $\geq 27$ weeks. $^{20}$

Household socioeconomic status was estimated using three proxy indices (housing quality, household assets and household livestock holdings), as previously described. ${ }^{22}$ Household food insecurity, defined as the limited or uncertain access to adequate food of sufficient quality, was assessed using the household food insecurity access categories. ${ }^{23}$ Pregnant women who reported consuming at least 5 of 10 defined food groups in the previous 24 hours (using a list-based food frequency questionnaire) were considered to have met the minimum dietary diversity for women (MDD-W) requirement. ${ }^{24}$

To control for the effect of season on study outcomes, each month 4-10 villages in the catchment area of a new IHC were visited for the baseline survey to enrol new participants monthly for 12 months. Once all the follow-up data were collected in the villages, the respective IHC was eligible for the programmatic intervention as described below. The endline survey was identical in structure and data collection strategies to those of the baseline survey. After the initial 12-month baseline period, the baseline survey continued in an additional six IHCs. The surveys were temporally staggered to ensure that there was a baseline village to compare with an endline village at each time point and allow time-matched comparisons between intervention and control villages. The latter six control IHCs did not benefit from the programmatic intervention described below but received donations of essential medications at the end of the project.

\section{Programmatic intervention}

Timing of surveys and the programmatic intervention

As soon as the baseline survey was completed in an IHC, the complete programmatic intervention was intended to start immediately in that IHC and its catchment area, and the endline survey was implemented 6 months after the completion of the baseline survey. Each month another IHC followed the same schedule (figure 1). However, due to various challenges encountered at different levels, the individual programmatic activities started with some delays (figure 2). The intervention consisted of three main strategies: (1) bimonthly behaviour change communication (BCC) activities in each participating village, (2) provision of essential drugs and supplies and (3) quality improvement (QI) activities at participating IHC, as previously described in more detail. ${ }^{16}$ The programmatic interventions were targeted at all pregnant women visiting 1 of the 12 participating intervention IHCs and/ or residing in participating intervention villages.

\section{Community-based BCC activities}

In each participating village, four community health workers (two men and two women) were identified in collaboration with the respective IHC to conduct BCC sessions. Selection criteria for these individuals included that they were already active volunteers in their village, that at least two among each group of four could read and write and that they were interested and available to conduct two BCC sessions per month.

During a 3-day training, the community health workers were trained in BCC, focused on the importance of adequate nutrition during pregnancy, IFA 
IHC1 IHC2 IHC3 IHC4 IHC5 IHC6 IHC7 IHC8 IHC9 IHC10 IHC11 IHC12

\begin{tabular}{|c|c|c|c|c|c|c|c|c|c|c|c|c|}
\hline $\begin{array}{l}\text { Community- } \\
\text { based behavior } \\
\text { change } \\
\text { communication } \\
\text { activities }\end{array}$ & $\Delta$ & $\Delta$ & $\Delta$ & $\Delta$ & $\square$ & $\square$ & $\square$ & $\square$ & 政 & 0 & . & $\square$ \\
\hline $\begin{array}{l}\text { Supplies and } \\
\text { drugs donation } \\
\text { to IHC }\end{array}$ & $\square$ & $\square$ & $\square$ & $\square$ & $\square$ & $\square$ & $\square$ & $\square$ & $\square$ & $\square$ & $\square$ & $\square$ \\
\hline $\begin{array}{l}\text { Quality } \\
\text { improvement } \\
\text { at IHC }\end{array}$ & $\mathrm{O}$ & $\mathrm{O}$ & $\mathrm{O}$ & 0 & $\Delta$ & $\Delta$ & $\Delta$ & $\Delta$ & $\square$ & $\square$ & $\square$ & $\square$ \\
\hline
\end{tabular}

Figure 2 Implementation of the selected components of the programmatic intervention. $\square$ Selected component of the intervention was implemented over 6 months. $\Delta$ Selected component of the intervention was implemented $<6$ months due to delays. O Selected component of the intervention was not implemented due to delays.

supplementation, the overall importance of ANC for the mother and the fetus and of seeking ANC early in the first trimester of pregnancy, prevention of malaria, recognition of danger signs during pregnancy, and the need for a birth plan. ${ }^{162526}$ Each community health worker received visual aids for use during the BCC activities. The BCC activities included discussion sessions and/or theatre performances with different target groups (men only, women only or both together) and the sites were opportunistic, and included gathering spaces such as the markets, mosques, schools and the houses of village chiefs. The applied theoretical framework of the BCC strategy is based on the recognition that individual behaviours are shaped by personal choices, social norms, environmental and economic constraints. ${ }^{27}$ The potential pathway of the BCC strategy was outlined in a programme impact pathway (online supplementary figure 1).

Provision of essential drugs and supplies

At the beginning of the present project, a facility assessment conducted in 12 IHCs identified large gaps in the availability of essential commodities and supplies. ${ }^{16}$ Thus, one of the intervention strategies was to procure and distribute needed drugs and supplies, which included IFA supplements, impregnated bednets, deworming medication, health cards for mothers and infants, cotrimoxazole, sulfadoxine pyrimethamine, amoxicillin, magnesium sulfate, malaria rapid diagnostic kits, urinalysis rapid diagnostic multistix, thermometers, stethoscopes, blood pressure monitors, scales and stadiometers. These donations were distributed to the selected IHCs in collaboration with the leadership of the regional and local health districts.

\section{Ql activities in the selected integrated health centers}

The implemented QI activities adopted a modified strategy of a previous quality assurance project conducted in Niger from 1993 to 1997, relying on formative supervision and coaching of key IHC personnel. ${ }^{28}$ Prior to the implementation of the programmatic intervention, a second programme impact pathway was developed for the health-centre level interventions (online supplementary figure 2). The present QI strategy was implemented in close collaboration with the regional and local health districts; one coach was identified in each of the two health districts and one at the regional level. All QI activities were implemented jointly by the project QI staff and the local QI coach. One training session was organised for health staff at participating IHC to explain the QI strategy and how to work together with the coaches towards improvements in ANC services.

After the initial training, the QI strategy was rolled out in each participating IHC as follows: first the QI team visited each IHC to identify difficulties related to the ANC activities. Identified difficulties were discussed with the IHC health staff and strategies for improvement were developed jointly. After 2-3 weeks, the QI team revisited the IHC for an evaluation and encouraged each IHC to further develop best solutions to solve their specific difficulties, and meetings occurred on a monthly basis thereafter.

\section{Operational research of programmatic intervention}

Over the course of the project implementation, operational research at multiple levels was used to assess programmatic activities. For community-based BCC activities, the community health workers' knowledge was assessed before and after the BCC training. In addition, a supervisory team used a structured form to monitor the content and the quality of each BCC session, which was used to provide constructive feedback to improve future sessions.

At IHCs, operational research was used to monitor QI activities and the availability of essential drugs and supplies. Direct observations of ANC sessions were conducted followed by postobservation interviews with pregnant women. Health agents in IHCs were also interviewed to assess the health centre's infrastructure including number of buildings, water and power supply, staff, and available drugs and supplies prior to, and over the course of, the project. 


\section{Sample size}

The sample size of the present study was designed to be able to detect a difference of $10 \%$ in the prevalence of anaemia between the baseline and endline surveys. In the absence of information on the magnitude of the intracluster correlation, the design effect was arbitrarily set at 2 to account for the cluster sampling design. Assuming an initial anaemia prevalence of $50 \%$, a significance level of 0.05 , power of 0.80 and a design effect of 2 , a sample size of 768 was needed, which was then inflated by $17 \%$ for attrition, yielding a target sample size of 925 pregnant women for both the baseline and endline survey. The baseline survey was extended concurrently with the endline survey for 6 months to serve as a pre-and-post and time-matched comparison and to account for the impact of the effect of the season of participants' enrolment on study outcomes. Based on the same assumption as above, the additional sample size required for extending the baseline survey was estimated at 77 pregnant women per IHC (ie, per month) resulting in a total of 463 over 6 months. Thus, in total 2313 pregnant women were required for the whole study (1388 baseline, 925 endline).

\section{Patient and public involvement}

Patients and the public were not involved in the design and the conduct of this study.

\section{Statistical analyses}

Data were double-entered using EpiData V.3.1. All statistical analyses were performed using the SAS System software V.9.4. Analyses were based on complete case intention-to-treat. Descriptive univariate statistics were calculated for all variables and variables not normally distributed (eg, GWG per week, MUAC and gestational age in weeks) were transformed to achieve a more normal distribution prior to analysis. Individual participant surveys (from each of the two time points-baseline and endline) were the unit of analysis. A detailed statistical analyses plan is available online. ${ }^{20}$

One primary outcome was attendance of an adequate number of ANC, defined as whether a woman reportedly participated in an adequate number of ANC visits at any health facility during her current pregnancy, based on her estimated gestational age. This was defined for the purposes of this study as: at least one reported ANC visit for women with a gestational age of 14-27 weeks, at least two reported ANC visits for women with a gestational age of 28-34 weeks, at least three reported ANC visits for women with a gestational age 35-39 weeks and at least four reported ANC visits for women with a gestational age of 40-42 weeks.

Other primary outcomes were reported adherence to the recommended IFA supplementation (ie, a woman reported consuming IFA supplements daily in the previous 7 days if she reported receiving any IFA supplements during her current pregnancy); knowledge of the benefits of IFA (ie, the woman reported knowledge of an illness where it is said that pregnant women do not have enough blood (local definition of anaemia) and responded that IFA supplements were used to prevent/ treat anaemia); and prevalence of anaemia $(\mathrm{Hb}<110 \mathrm{~g} / \mathrm{L}$ in the first and third trimesters and $\mathrm{Hb}<105 \mathrm{~g} / \mathrm{L}$ in the second trimester). ${ }^{4}$ Secondary outcomes were: mean GWG per week (maternal weight measured $~ 1$ month apart and GWG calculated as the difference between measurements at visit 2 and visit 1 divided by number of elapsed days and multiplied by 7); mean MUAC and mean $\mathrm{Hb}$ concentration; prevalence of adequate GWG (defined as a GWG of $0.35-0.58 \mathrm{~kg} /$ week, using the US Institute of Medicine recommendations for underweight and normal weight women combined) and adequate MUAC (MUAC $\geq 23 \mathrm{~cm}$ ). ${ }^{29}$

Because the BCC activities were implemented at the village level, the village was considered the cluster in the present study. Twenty-four of the 92 villages had small numbers of participants (ie, $<5$ women). To avoid unstable estimates due to small sample sizes per cluster, villages with $<5$ participants were combined with villages of $>5$ participants and which had similar characteristics such as belonging to the same IHC, and located approximatively at the same distance from the IHC. Consequently, the analyses included 68 clusters.

The study was designed such that the baseline survey continued during the endline survey. A preintervention and postintervention analysis comparing the baseline cohort to the endline cohort was performed, and time of assessment was considered in the analysis by including year and season in all models as described in more detail below. The PROC GLIMMIX procedure in SAS was performed to estimate the adjusted OR (for binary outcomes) or the adjusted mean difference (for continuous outcomes). A p value $<0.05$ was considered statistically significant. In order to account for the cluster design of this quasi-experimental design, robust SEs were used in all models, using the EMPIRICAL=FIRORES option in PROC GLIMMIX, with random intercepts specified for each cluster via a RANDOM statement. The level of randomisation for the robust SEs is the village. Both minimally and fully adjusted models were used in the analysis. Minimally adjusted models included year, season and village to account for the effect of season on the study outcomes. Covariates in these analyses included age of the pregnant women as a binary variable (adolescent ( $\leq 19$ years old) vs adult ( $>19$ years old)), ethnicity (Hausa vs other minority ethnic groups), woman's principal occupation (housewife vs non-housewife), marital status (married vs separated/divorced or widow), gravidity (primigravida vs multigravida), age at first pregnancy (as continuous variable), outcome of last pregnancy (living child vs child not born alive / born alive and had since died), attended any ANC during last pregnancy (yes vs no), attended at least four ANC during last pregnancy (yes vs no), health facility delivery during last pregnancy (yes vs no), household head's education level (some primary education or higher vs no education / literacy training only / koranic school), principal occupation of the household head 


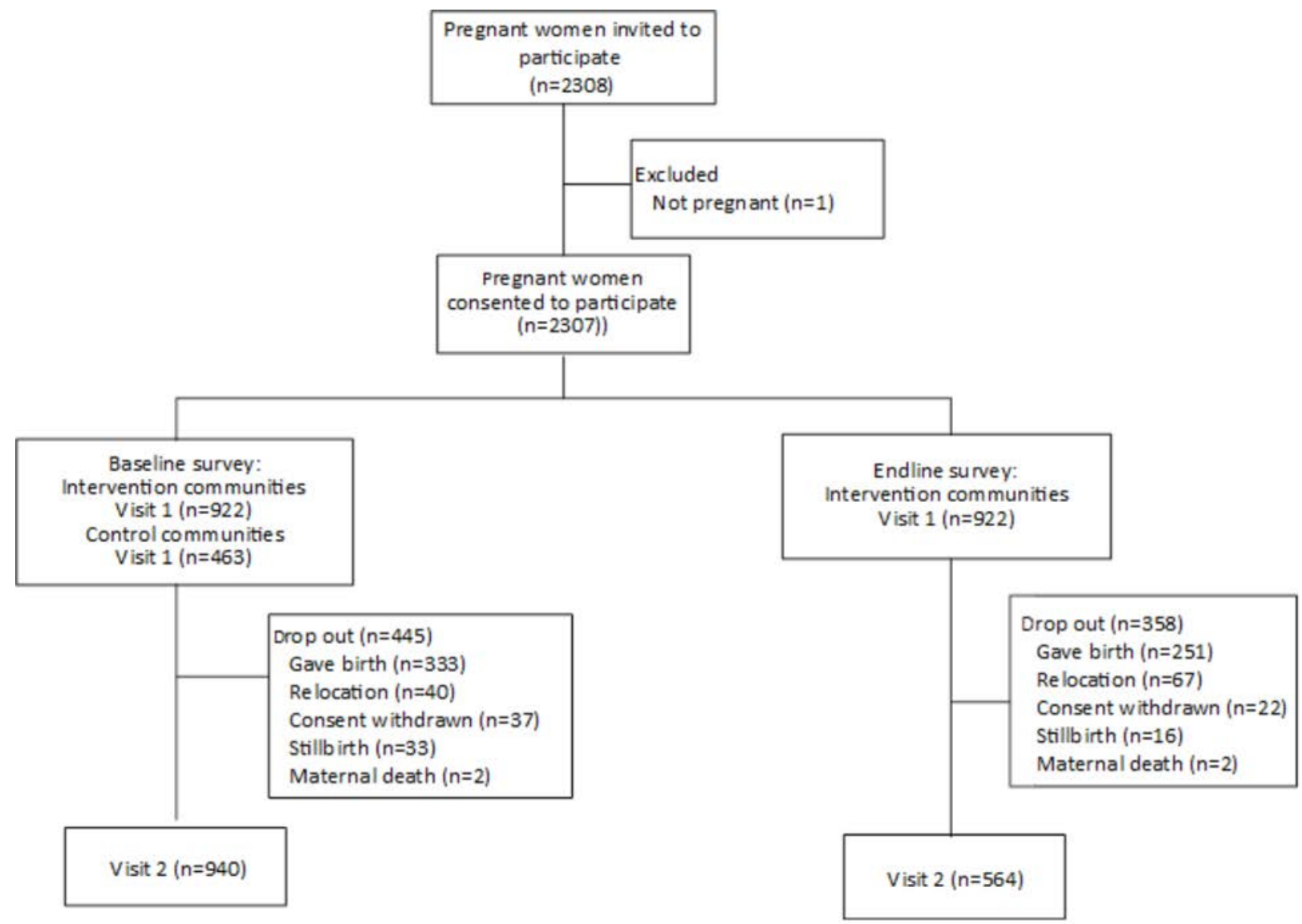

Figure 3 Flow diagram of participants through the baseline and endline surveys.

(farming-related occupation vs non-farming-related occupation), level of household food insecurity (food secure vs mildly / moderately / severely food insecure). Fully adjusted models included all the covariates in addition to year, season, village and a variable indicating if a participant was enrolled in the baseline or in the endline survey.

We also examined whether specific demographic characteristics (eg, maternal age, education, occupation, housing quality, household assets, household livestock, reported danger signs during current pregnancy, last pregnancy outcome, quantity of food consumed per day compared with the time when the woman was not pregnant, number of meals per day, age of gestation) were effect modifiers to the impact on all the outcomes, using interaction terms.

\section{RESULTS}

\section{Initial characteristics of study population}

A total of 2307 pregnant women from 68 clusters were enrolled for both baseline and endline surveys. All women who were invited to participate in the study agreed to participate and provided written informed consent. Of the women who participated in visit 1 from either the baseline or endline surveys, $67.9 \%(\mathrm{n}=940)$ and $61.7 \%(\mathrm{n}=564)$ completed visit 2 , respectively (figure 3 ). The primary reason for loss to follow-up was childbirth.
The majority of pregnant women $(83.7 \%$ and $78.5 \%$ at baseline and endline, respectively) were adults (age $>19$ years). Most participants $(79.1 \%$ and $78.6 \%$ at baseline and endline surveys, respectively) had not received any formal education and most of them reported to be housewives as their primary profession. Women who were enrolled in the baseline survey differed from those participating in the endline survey in a few of their personal and household characteristics, such as the proportion of adolescent women, health facility delivery during last pregnancy, level of household food insecurity, principal occupation of the household head and season of enrolment in the survey (table 1).

In addition, some characteristics differed between women who were lost to follow-up and women who completed visit 2. At the baseline survey, adult women $(\mathrm{p}=0.009)$, housewives $(\mathrm{p}=0.01)$, women in the third trimester of gestation $(\mathrm{p}<0.0001)$ and those who attended fewer than 4 ANC during their last pregnancy ( $p=0.046)$ were more likely to be lost to follow-up. At the endline survey, women in the third trimester of gestation $(\mathrm{p}<0.0001)$, those who delivered at a health facility during their last pregnancy $(p=0.006)$ and those living with a household head whose principal occupation was not farming $(\mathrm{p}=0.04)$ were more likely to be lost to follow-up. 
Table 1 Characteristics of pregnant women in the baseline versus endline surveys ${ }^{*}$

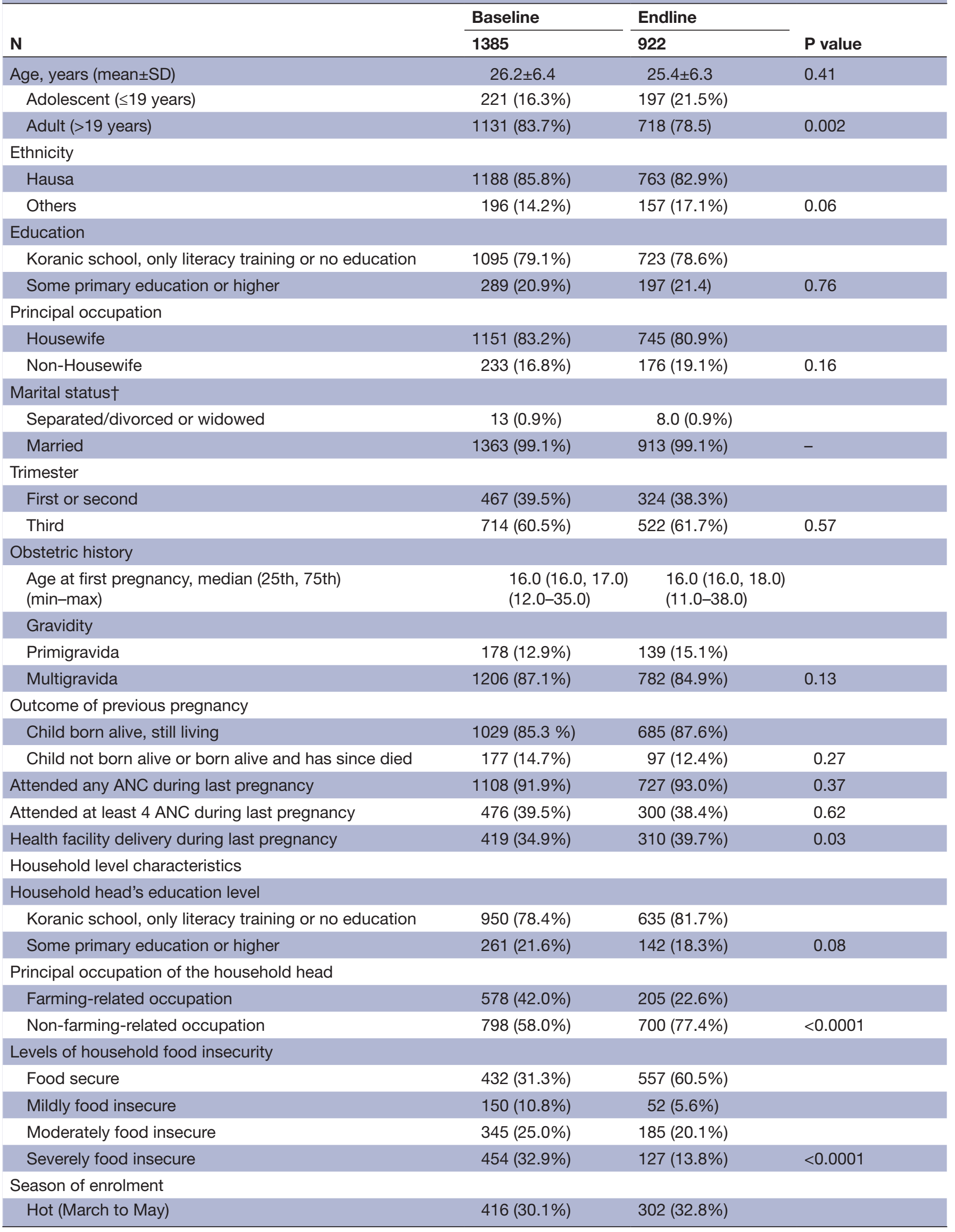




\begin{tabular}{|c|c|c|c|}
\hline & Baseline & Endline & \\
\hline $\mathbf{N}$ & 1385 & 922 & $P$ value \\
\hline Raining (June to September) & $501(36.2 \%)$ & $275(29.9 \%)$ & \\
\hline Dry (October to February) & $467(33.7 \%)$ & $344(37.3 \%)$ & 0.007 \\
\hline
\end{tabular}

${ }^{*}$ Results are presented as $\mathrm{N}(\%)$ or mean $\pm \mathrm{SD}$, unless stated otherwise. †Categories were too small to calculate a $p$ value.

ANC, antenatal care; max, maximum; min, minimum.

\section{Effects of the programmatic intervention \\ ANC attendance}

Across all IHCs, after 6 months of intervention, the proportion of pregnant women who reportedly attended any ANC during the current pregnancy was not statistically different between baseline and endline surveys $(73.9 \%$ vs $77.4 \%$, endline vs baseline OR: $1.5(0.7,3.2)$; table 2).

A low proportion of women attended ANC during their first trimester of gestation, and was not significantly different between baseline and endline surveys. The percentage of pregnant women who reported attending a gestational age-specific adequate number of ANC was also not significantly different between the baseline and the endline survey $(63.8 \%$ vs $59.1 \%$, endline vs baseline OR: $1.0(0.5,2.0))$.

IFA supplementation and $\mathrm{Hb}$ concentration

Multivariable analyses showed that women participating in the endline survey were more likely to know what an illness is, where it is said that pregnant women do not have enough blood (that we described as anaemia), $(81.4 \%)$ than those in the baseline survey $(43.1 \%$, OR : $5.7(2.5,13.3))$. Among those who knew what anaemia was, women participating in the endline survey were more likely to know the benefits of IFA $(65.0 \%)$ than those in the baseline survey $(53.2 \%$, OR $4.1(1.7,9.5))$. In addition to the knowledge of anaemia and the benefit of IFA, pregnant women in the endline survey were more likely to have received any amount of IFA supplements during their current pregnancy $(60.0 \%)$ than the participants in the baseline survey (45.8\%, endline vs baseline OR: 2.6 $(1.2,5.4)$; table 3$)$ and this difference remained significant in the fully adjusted analyses (OR: $2.7(1.2,6.1)$ ).

Among women who reported receiving IFA in their current pregnancy, the proportion of those who reported consuming IFA daily the week before the interview did not differ significantly from the endline survey compared with the baseline survey $(77.3 \%$ vs $71.8 \%$, $\mathrm{p}=0.54$ ). However, the overall percentage of women both receiving and consuming IFA daily in the last 7 days as recommended was higher in the endline survey than in the baseline survey $(46.4 \%$ vs $32.8 \%$, OR: $2.8(1.2,6.5)$; figure 4 and table 3$)$. There was a significant interaction between the effect of the intervention on the adherence of IFA supplementation and housing quality (a proxy of socioeconomic status) in both the minimally adjusted

Table 2 Comparison of reported antenatal care seeking in the baseline versus endline surveys

\begin{tabular}{|c|c|c|c|c|c|c|}
\hline & $\begin{array}{l}\text { Baseline } \\
(n=1385)\end{array}$ & $\begin{array}{l}\text { Endline } \\
(\mathrm{n}=922)\end{array}$ & $\begin{array}{l}\text { Minimally adjusted } \\
\text { OR or minimally } \\
\text { adjusted mean } \\
\text { difference* }(95 \% \mathrm{Cl})\end{array}$ & $P$ value & $\begin{array}{l}\text { Fully adjusted OR or } \\
\text { fully adjusted mean } \\
\text { difference } † \\
(95 \% \mathrm{Cl})\end{array}$ & $P$ value \\
\hline \multicolumn{7}{|c|}{ Attended any ANC during the current pregnancy } \\
\hline Yes & $1023(73.9 \%)$ & $713(77.4 \%)$ & 1.5 (0.8 to 2.8$)$ & 0.18 & 1.5 (0.7 to 3.2$)$ & 0.32 \\
\hline No & $361(26.1 \%)$ & $208(22.6 \%)$ & Ref & & Ref & \\
\hline \multicolumn{7}{|c|}{ Attended ANC in the first trimester of gestation $\neq \S$} \\
\hline Yes & $41(4.1 \%)$ & $25(3.6 \%)$ & 0.2 & - & 1.0 & - \\
\hline No & 967 (95.9\%) & $671(96.4 \%)$ & Ref & & Ref & \\
\hline \multicolumn{7}{|c|}{ Attended adequate number of ANC for gestational age } \\
\hline Yes & $644(63.8 \%)$ & $416(59.1 \%)$ & 0.7 (0.4 to 1.4$)$ & 0.32 & $1.0(0.5$ to 2.0$)$ & 0.92 \\
\hline No & 366 (36.2\%) & 288 (40.9\%) & Ref & & Ref & \\
\hline
\end{tabular}

*Minimally adjusted model: adjusted for year, season, village and a variable indicating if a participant was enrolled in the baseline or in the endline survey because of the nature of the study design.

tFully adjusted model: multivariable logistic regression, adjusted for all the covariates in addition to year, season, village and a variable indicating if a participant was enrolled in the baseline or in the endline survey.

$\ddagger$ Categories were too small to calculate a $p$ value.

§Among pregnant women who reported having attended any ANC, $n=1008$ in the baseline survey and $n=696$ in the endline survey.

ПAmong pregnant women who reported having attended any ANC, $n=1010$ in the baseline survey and $n=704$ in the endline survey.

ANC, antenatal care. 
Table 3 Comparison of iron folic acid supplementation and haemoglobin concentration in the baseline versus endline surveys*

\begin{tabular}{|c|c|c|c|c|c|c|}
\hline \multirow[b]{2}{*}{ IFA supplementation, $\mathbf{n}$} & \multirow{2}{*}{$\begin{array}{l}\text { Baseline } \\
1385\end{array}$} & \multirow{2}{*}{$\begin{array}{l}\text { Endline } \\
922\end{array}$} & \multirow{2}{*}{$\begin{array}{l}\text { Minimally adjusted OR or } \\
\text { minimally adjusted mean } \\
\text { difference }(95 \% \mathrm{Cl})\end{array}$} & \multirow[b]{2}{*}{$P$ value } & \multirow{2}{*}{$\begin{array}{l}\text { Fully adjusted OR or fully } \\
\text { adjusted mean difference } \\
(95 \% \mathrm{Cl})\end{array}$} & \multirow[b]{2}{*}{$P$ value } \\
\hline & & & & & & \\
\hline \multicolumn{7}{|l|}{ Knowledge of anaemia } \\
\hline Yes & $596(43.1 \%)$ & $744(81.4 \%)$ & $5.4(2.5$ to 11.8$)$ & $<0.0001$ & 5.7 (2.5 to 13.3$)$ & 0.0001 \\
\hline No & $787(56.9 \%)$ & $170(18.6 \%)$ & Ref & & Ref & \\
\hline \multicolumn{7}{|l|}{ Knew benefits of IFA§ } \\
\hline Yes & $316(53.2 \%)$ & $484(65.0 \%)$ & $3.2(1.6$ to 6.5$)$ & 0.001 & 4.5 (1.9 to 10.6$)$ & 0.0009 \\
\hline No & $278(46.8 \%)$ & $259(35.0 \%)$ & Ref & & Ref & \\
\hline \multicolumn{7}{|c|}{ Reportedly received IFA during current pregnancy } \\
\hline Yes & $633(45.8 \%)$ & $552(60.0 \%)$ & $2.6(1.2$ to 5.4$)$ & 0.01 & $2.7(1.2$ to 6.1$)$ & 0.02 \\
\hline No & $748(54.2 \%)$ & $368(40.0 \%)$ & Ref & & Ref & \\
\hline \multicolumn{7}{|c|}{ Reportedly adhered to IFA supplementation } \\
\hline Yes & $455(32.8 \%)$ & $428(46.4 \%)$ & 2.4 (1.1 to 5.3$)$ & 0.04 & 2.8 (1.2 to 6.5$)$ & 0.02 \\
\hline No & $930(67.2 \%)$ & $494(53.6 \%)$ & Ref & & Ref & \\
\hline Anaemia, $n$ & 920 & 555 & & & & \\
\hline Haemoglobin (g/L)ף & $96.7 \pm 2.2$ & $95.6 \pm 2.5$ & $-0.1(-0.5$ to 0.3$)$ & 0.60 & $-0.2(-0.6$ to 0.2$)$ & 0.30 \\
\hline \multicolumn{7}{|l|}{ Anaemia prevalence ${ }^{\star \star}$} \\
\hline Anaemic & $724(79.5 \%)$ & $426(78.2 \%)$ & $1.2(0.6$ to 2.8$)$ & 0.60 & $1.4(0.6$ to 3.6$)$ & 0.41 \\
\hline Non-anaemic & $187(20.5 \%)$ & $119(21.8 \%)$ & Ref & & Ref & \\
\hline
\end{tabular}

${ }^{*}$ Results are presented as $\mathrm{N}(\%)$ or mean \pm SE.

†Minimally adjusted model: adjusted for year, season, village and a variable indicating if a participant was enrolled in the baseline or in the endline survey because of the nature of the study design.

fFully adjusted model: multivariable logistic regression, adjusted for all the covariates in addition to year, season, village and a variable indicating if a participant was enrolled in the baseline or in the endline survey.

$\S$ Among pregnant women, who responded that they knew anemia, locally known as 'not having enough blood', $n=594$ in the baseline survey and $\mathrm{n}=743$ in the endline survey.

१The mean difference compares endline to baseline.

${ }^{* *}$ Anemia is defined as haemoglobin concentration $<110 \mathrm{~g} / \mathrm{L}$ if first and third trimester of gestation; $<105 \mathrm{~g} / \mathrm{L}$ if second trimester of gestation.

IFA, iron folic acid.

$(\mathrm{p}=0.009)$ and the fully adjusted models $(\mathrm{p}=0.0006)$. Pregnant women living in households with a housing quality index above the median were more likely to report adherence to recommended IFA supplementation $(52.1 \%)$ than those with a housing quality index at or below the median $(38.2 \%)$ (minimally adjusted OR: 3.7 $(1.3,10.3)$, fully adjusted OR: $5.2(1.6,16.7))$.

Housing quality $(\mathrm{p}=0.007)$ and maternal age $(\mathrm{p}=0.02)$ modified the effect of the intervention on the knowledge of IFA benefits. In the fully adjusted analyses, the interaction remained statistically significant for housing quality $(\mathrm{p}=0.02)$, and was marginally significant for maternal age $(p=0.07)$. After the intervention, pregnant women living in the households with a housing quality index at or below the median $(71.0 \%)$ were more likely to know the benefits of IFA supplementation than those with a housing quality index above the median $(60.9 \%$; OR: 2.18, $\mathrm{p}<0.0001$; minimally adjusted OR: 4.1 (1.4, 12.0), $\mathrm{p}=0.001$; fully adjusted OR: $5.4(1.4,20.4), \mathrm{p}=0.002)$. In addition, adult pregnant women were more likely to report knowing the benefits of IFA supplementation $(67.2 \%)$ compared with adolescent pregnant women (56.6\%; minimally adjusted OR: $3.6(1.8,7.2), \mathrm{p}=0.0008$; fully adjusted OR: $4.2(1.7,10.4), \mathrm{p}=0.02)$.
The mean $\mathrm{Hb}$ concentrations of pregnant women in the baseline survey and endline surveys were not statistically different (table 3). There was also no statistically significant difference between anaemia prevalence among pregnant women at the baseline survey compared with those at the endline survey.

\section{GWG, MUAC and MDD-W}

The mean GWG and mean MUAC, as well as the prevalence of adequate GWG per week, and adequate MUAC were not statistically different between women participating in the baseline compared with the endline survey (table 4).

There was also no statistically significant difference between the prevalence of adequate MDD-W among pregnant women at the endline survey $(30.6 \%)$ compared with those at the baseline survey $(25.3 \%$, minimally adjusted OR: $1.6(0.7,3.7)$, fully adjusted OR: $1.4(0.5,3.3))$.

Community-based BCC activities, ANC visit observation and infrastructure survey

Over the course of the project, 233 community health workers were trained in and conducted the communitybased BCC activities. In the endline survey, $32.7 \%$ of 

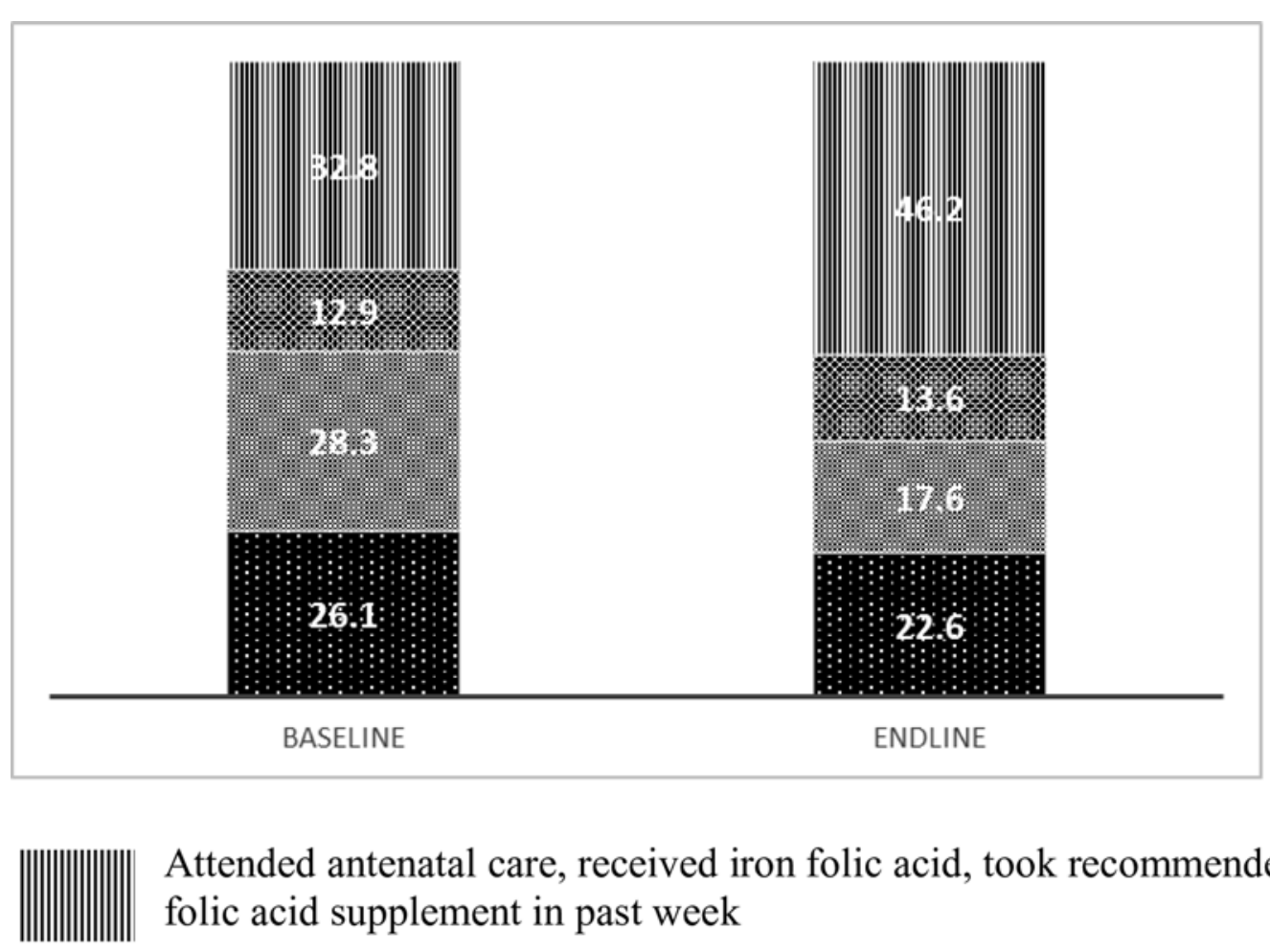

\section{Attended antenatal care, received iron folic acid, took recommended number of iron folic acid supplement in past week}

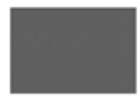

Attended antenatal care, received iron folic acid, did not took recommended amount of iron folic acid supplement in past week

\section{Attended antenatal care, did not receive iron folic acid supplements}

\section{Did not attend antenatal care}

Figure 4 Percentage of pregnant women who reported attending antenatal care, receiving iron folic acid and adhering to the recommended iron folic acid supplementation in the baseline versus endline surveys.

Table 4 Comparison of gestational weight gain and mid-upper arm circumference for women in the baseline versus endline surveys*

\begin{tabular}{|c|c|c|c|c|c|c|}
\hline Characteristics & Baseline & Endline & $\begin{array}{l}\text { Minimally adjusted OR or } \\
\text { minimally adjusted mean } \\
\text { difference }(95 \% \mathrm{Cl})\end{array}$ & $P$ value & $\begin{array}{l}\text { Fully adjusted OR or fully } \\
\text { adjusted mean difference } \\
(95 \% \mathrm{Cl})\end{array}$ & $P$ value \\
\hline $\begin{array}{l}\text { Gestational weight gain } \\
\text { (GWG), n }\end{array}$ & 940 & 564 & & & & \\
\hline Adequate GWG per week§ & $224(24.0 \%)$ & $148(26.4 \%)$ & 0.5 (0.2 to 1.2$)$ & 0.13 & 0.5 (0.2 to 1.5$)$ & 0.22 \\
\hline GWG per week (kg)ף & $0.20 \pm 0.07$ & $0.28 \pm 0.07$ & $0.07(-0.04$ to 0.19$)$ & 0.21 & $0.06(-0.07$ to 0.19$)$ & 0.36 \\
\hline MUAC, $n$ & 1385 & 922 & & & & \\
\hline Adequate MUAC ( $\geq 23 \mathrm{~cm})$ & $1041(75.2 \%)$ & $712(77.2 \%)$ & $1.3(0.7$ to 2.5$)$ & 0.38 & 1.4 (0.6 to 3.2$)$ & 0.41 \\
\hline $\operatorname{MUAC}(\mathrm{cm})$ ๆ & $24.7 \pm 0.27$ & $24.3 \pm 0.33$ & $-0.4(-1.0$ to 0.2$)$ & 0.24 & $-0.3(-1.0$ to 0.3$)$ & 0.34 \\
\hline
\end{tabular}

${ }^{*}$ Results are presented as $\mathrm{N}(\%)$ or mean \pm SE.

†Minimally adjusted model: adjusted for year, season, village and a variable indicating if a participant was enrolled in the baseline or in the endline survey because of the nature of the study design.

fFully adjusted model: multivariable logistic regression, adjusted for all the covariates in addition to year, season and village.

§Adequate gestational weight gain per week compared with the Institute of Medicine recommendations for underweight and normal weight women: a GWG of $0.35-0.58 \mathrm{~kg} /$ week was considered adequate. ${ }^{41}$

ITThe mean difference compares endline to baseline.

GWG, gestational weight gain; MUAC, mid-upper arm circumference. 
pregnant women reported having participated in some community BCC activities. Of those who participated in the community activities in their village, half of them attended more than one session. BCC activities were also open to other community members, and $24.1 \%$ of women in the endline survey reported that at least one of their family members had participated in at least one of these education sessions. Among the women who did not participate, $68.2 \%$ reported that they did not know that there were education sessions in their village and $21.3 \%$ reported that there were no educational sessions in their village in the previous 6 months.

As part of operational research, ANC consultations at participating IHC were observed for 49 pregnant women before the start of the intervention, and for 89 pregnant women at the end of the intervention. Before the intervention, signs and symptoms of anaemia including fatigue and pallor (conjunctival and/or palmor) were assessed by health agent in charge of ANC visit among $25.5 \%$ and $95.7 \%$ pregnant women, respectively. At the end of the intervention, the aforementioned signs and symptoms of anaemia were assessed among $68.5 \%$ and $95.5 \%$ pregnant women, respectively. IFA supplements were given to $55.3 \%$ of pregnant women before the start of the intervention and to $98.8 \%$ of pregnant women at the end of the intervention suggesting that the distribution of essential supplies resulted in higher IFA distribution. Over the course of the programmatic intervention infrastructure surveys were conducted in eight participating IHCs. Before the intervention, seven out of eight IHCs had IFA in stock and all eight IHCs had IFA in stock at the end of the intervention.

\section{DISCUSSION}

This study aimed to assess the impacts of a set of programmatic intervention consisting of three main strategies: (1) bimonthly community-based BCC activities in each participating village, (2) provision of essential drugs and supplies to IHCs and (3) QI activities at participating IHCs. Our results indicate that the programmatic intervention did not have an impact on overall ANC seeking and did not affect the percentage of pregnant women who reported attending the adequate number of ANC based on their estimated gestational age. In contrast, a preintervention and postintervention comparison study in Uganda where the intervention included community mobilisation and empowerment to stimulate demand for services (ie, home visits by community health workers, health education through radio spots, talk shows and quarterly community dialogues, promotion of saving, and promotion of partnerships with local transporters to facilitate geographical access to care); and health provider capacity building (ie, emergency obstetric and newborn care refresher training, mentorship and support supervision of primary health workers, certificate courses in health services management and project planning, and recognition of best performing facilities and managers) found a significant increase in the proportion of pregnant women who attended at least four ANC visits. ${ }^{30}$ Our intervention also failed to increase the proportion of women who attended ANC early in their pregnancy. This is in contrast to the study in Uganda described above, as well as a precomparison and postcomparison study in Tanzania, where the proportion of primigravida who attended ANC visits between 4 and 16 weeks of gestation significantly increased from $19 \%$ to $57 \%$ after 2 years of a community-based safe motherhood intervention promoting early ANC, the recommended number of ANC visits and delivery with a skilled attendant. ${ }^{30} 31$ The determinants of the use of ANC services are multifactorial and complex, including demographic characteristics, socioeconomic factors, predisposing cultural and religious factors, social support, factors related to healthcare providers, women's knowledge and attitude and medical or obstetric history, potentially explaining the different outcomes between the present study and the aforementioned studies in Uganda and Tanzania. ${ }^{22}{ }^{32}$ In addition, in our study, the intervention was implemented for only 6 months, which was likely too short to generate a substantial behavioural change. A longer and more sustained intervention may have shown a broader impact of the programmatic intervention.

In the present study, the proportion of pregnant women who reported receiving IFA supplements during their current pregnancy was significantly higher in the endline survey compared with the baseline survey. This may be unexpected finding considering that there was no impact on ANC attendance. An explanation of the increase of the proportion of women reported receiving IFA supplements at during endline survey may be due to QI at each intervention health centre and BCC activity at village level. The QI, which included refresher training in ANC skills for health agents (in knowledge and counselling techniques) and coaching/supportive supervision of health staff may have led to improvements in skills and competence, and in counselling techniques of health agents and resulted in distribution of IFA to women receiving during ANC. Consequently, the proportion of pregnant women who reported attending ANC, and receiving and consuming the recommended number of IFA supplements in the week prior the interview was higher at endline versus baseline. These results were supported by our findings from operational research, where we observed that more pregnant women received IFA during ANC after the programmatic intervention had been implemented. This was likely due to the donation of IFA supplements to IHCs and the QI activities. Similar to the present study, a quasi-experimental study among pregnant women in rural India, with the aim to enhance dietary and IFA intake, also showed an increased proportion of women who consumed IFA supplements for 100 days after only 12 weeks of an intervention, which consisted of personal communication between the interviewer and the participant, as well as home-based reminder materials. ${ }^{33}$ 
The present study showed that the proportion of pregnant women who reported knowing the benefits of IFA supplementation was significantly higher in the endline survey than in the baseline survey, especially among adult pregnant women and those with housing index at or below the median (proxy for low socioeconomic status). It has been shown that in low-income countries pregnant women use their relatives, especially mothers and mothers-in-law as their primary source of information for pregnancy, postnatal health, parenting, breastfeeding, immunisation programme and family planning. ${ }^{34}$ However, we do not know why pregnant women with low socioeconomic status were more likely to report knowing the benefits of IFA. The programmatic intervention provided different channels of communication (BCC activities in the community or ANC consultation) where women could improve their knowledge. Given there was no overall improvement in women's ANC attendance, we assume that the increase of the proportion of women knowing the benefit IFA was due to the BCC activities. In addition, because IFA was more available and more often distributed by health staff in the IHCs, the benefits of IFA might also have been discussed with women who attended the ANC visits.

The present study did not show a statistically significant impact of the intervention on mean $\mathrm{Hb}$ concentration or on anaemia prevalence. Our findings are similar to a study in Senegal, where an intervention including the promotion of iron supplementation during pregnancy and nutrition education to increase intake of iron-rich foods did not show an impact on $\mathrm{Hb}$ concentration. ${ }^{35} \mathrm{In}$ contrast, studies in India and Sri Lanka have shown an increase in $\mathrm{Hb}$ concentration after educational interventions alone. ${ }^{3637}$ These differences may be due to differences in the types of interventions. First, the study in India consisted of an intense educational intervention with weekly home visits to reinforce educational messages, listen to participants' problems and check compliance with IFA supplementation. In Sri Lanka, the intervention consisted of an interactive educational session involving small groups of pregnant women during the recruitment. The educational messages focused on how to take the iron supplement to improve its bioavailability. In contrast, our study combined health-center-based intervention, including the donation of IFA supplements, with community BCC sessions, where IFA supplementation, increased intake of iron-rich foods, and the prevention of malaria during pregnancy were promoted. The operational research confirmed that IFA supplements were available in almost all IHC and were distributed by health staff over the course of the programmatic intervention. However, despite providing IFA supplements to health centres and increasing the proportion of women who received them, daily consumption of supplements in the week prior to the interview remained low. Thus, strategies to improve the compliance with IFA supplementation, for example, household visits to reinforce educational messages, monitor compliance and resolve barriers to supplementation consumption may need to be considered. Moreover, considering the high prevalence of anaemia and the previously reported high prevalence of deficiencies in multiple micronutrients in this study population, ${ }^{38}{ }^{39}$ it may not be adequate to resolve nutritional deficiencies with IFA supplements alone, and multiple micronutrients supplementation should be considered for this population.

The prevalence of inadequate GWG and low MUAC was high, indicating that undernutrition is a serious problem in the study population, but our study did not find a statistically significant impact of the intervention on pregnant women's GWG and MUAC. Similar to our findings, no impact was found on maternal weight gain after programmatic interventions in India and Cambodia. ${ }^{3640}$ Determinants of GWG include a range of maternal biological (eg, age, parity, stature and genetics), metabolic and social factors (eg, socioeconomic status, education, physical activity and diet). ${ }^{41}$ Therefore, it is possible that the present programmatic intervention did not target the right modifiable determinants or was not frequent and long enough. For instance, many pregnant women in our study lived in food-insecure households suggesting that balanced energy and protein supplementation may be necessary to achieve adequate GWG and to improve pregnancy outcomes. ${ }^{42}{ }^{43}$ Finally, it is possible that assessing GWG based on only two measurements over 1 month was inadequate to determine an impact on anthropometric outcomes.

The present study has a number of strengths including staggered enrolment over the course of a full year which allowed us to control for the potential effects of the season on the study outcomes. Moreover, this study included extensive data collection by highly trained and supervised field workers in a previously understudied population. Therefore, the study fills a significant gap in knowledge related to pregnant women's nutrition and health status in Niger. However, the present study has also several limitations. First, because health districts were selected based on convenience sampling, our sample of pregnant women is not representative of the population of the Zinder region nor of Niger. Despite using the random walk method and the help of a local guide, the recruitment process may have missed eligible women because of the time of day the recruitment was made or some women at the early stage of the pregnancy were not willing to disclose the pregnancy. Second, the study is a predesign and postdesign comparing a cohort of women at baseline to another cohort of women at endline, which is subject to internal validity threats associated with uncontrolled changes over time, including withinvillage developments and historical effects present in one comparison period but not the other. That said, this bias was reduced by controlling for year and season in all the models. In addition, there could be selection biases arising from differences in the characteristics of women in the two surveys. However, this is less likely a concern because all women who were invited to participate in the 
study provided consent. Because the present study relied on self-reported information, social desirability bias may be another concern. Although we were able to verify the reported ANC visits with the documented dates in the pregnant women's health booklet, we were not able to verify reports of IFA adherence. Ideally, the social desirability among the study participants should be assessed during data collection, ${ }^{44}{ }^{45}$ which was not the case in the present study. However, more women reported receiving IFA during ANC in the endline survey, which was also reflected in a higher rate of IFA distribution during observed ANC consultations. Various challenges also encountered during the study resulted in some delays in the implementation of the different programmatic activities. Subanalyses of villages that received the full set of intervention activities did not indicate any additional benefits (results not shown). Finally, the 6-month intervention period may not have been long enough to show an impact on the outcomes. However, despite the short duration of the intervention and the partial implementation of some of the intervention components due to some delays, we found a modest improvement on the adherence to IFA supplementation. Thus, a larger impact may be possible if the programmatic intervention using multiple strategies were to be implemented for a longer period.

\section{CONCLUSION}

The programmatic intervention resulted in a modest increase in the proportion of women who reported having received and consumed IFA. There was no statistically significant impact of the programmatic intervention on overall ANC service-seeking behaviour, or on the prevalence of anaemia, GWG, or adequate MUAC. The lack of positive impacts might be partially due to the short time of the intervention (6 months) and relative low intensity of the components of the intervention. However, in view of the high prevalence of inadequate GWG and low MUAC, more intensive interventions, balanced energy and protein dietary supplementation and/or multiple micronutrient supplementation may be required in the study population as recommended by WHO for undernourished populations. ${ }^{46}$

Acknowledgements We are grateful to the entire study team, including the data collection and data entry teams and other support staff. Our sincere appreciation goes to Zinder and Mirriah health districts and the national steering committee. We thank Daniel J Tancredi for his statistical guidance, and his and Stephen A Vosti's (both at the University of California, Davis, USA) valuable comments on the draft manuscript. Finally, we sincerely appreciate the support of the participants, the local communities and the community health workers. We further thank UNICEF, Niger, for the supply donation.

Contributors The NiMaNu Project was conceived and designed by SYH, KRW and CTO. Data were analysed by CTO with the guidance from RRY and SYH. Results were interpreted by all coauthors. CTO drafted the manuscript, and SYH edited the manuscript. All authors reviewed and approved the final version of the manuscript.

Funding This study was funded by the Government of Canada through Global Affairs Canada and Nutrition International. Nutriset SAS provided financial support for tuition fees of CTO's PhD dissertation.

Competing interests BN works for Nutrition International.

\section{Patient consent for publication Not required.}

Provenance and peer review Not commissioned; externally peer reviewed.

Data availability statement The datasets generated and analysed during the current study are available from the study principal investigator on reasonable request (syhess@ucdavis.edu).

Open access This is an open access article distributed in accordance with the Creative Commons Attribution Non Commercial (CC BY-NC 4.0) license, which permits others to distribute, remix, adapt, build upon this work non-commercially, and license their derivative works on different terms, provided the original work is properly cited, appropriate credit is given, any changes made indicated, and the use is non-commercial. See: http://creativecommons.org/licenses/by-nc/4.0/.

\section{ORCID iD}

Cesaire T Ouedraogo http://orcid.org/0000-0003-0634-3226

\section{REFERENCES}

1. The Partnership for Maternal, Newborn and Child Health. Opportunities for Africa's newborns: Practical data, policy and programmatic support for newborn care in Africa. Geneva World Health Organization; 2006.

2. Ghosh-Jerath S, Devasenapathy N, Singh A, et al. Antenatal care (ANC) utilization, dietary practices and nutritional outcomes in pregnant and recently delivered women in urban slums of Delhi, India: an exploratory cross-sectional study. Reprod Health 2015;12.

3. World Health Organization. Guideline: daily iron and folic acid supplementation in pregnant women. Geneva, Switzerland: World Health Organization, 2012

4. World Health Organization. WHO recommendations on antenatal care for a positive pregnancy experience. Geneva, Switzerland: World Health Organization, 2016.

5. Institut National de la Statistique (INS), ICF International. Enquête démographique et de santé et indicateurs multiples du Niger 2006. Calverton, Maryland, USA: INS et ICF International, 2007.

6. Finlayson K, Downe S. Why do women not use antenatal services in low- and middle-income countries? A meta-synthesis of qualitative studies. PLoS Med 2013;10:e1001373.

7. Pell C, Meñaca A, Were F, et al. Factors affecting antenatal care attendance: results from qualitative studies in Ghana, Kenya and Malawi. PLoS One 2013;8:e53747.10.1371/journal.pone.0053747

8. Andrew EVW, Pell C, Angwin A, et al. Factors affecting attendance at and timing of formal antenatal care: results from a qualitative study in Madang, Papua New Guinea. PLoS One 2014;9:e93025.

9. Mbuagbaw L, Medley N, Darzi AJ, et al. Health system and community level interventions for improving antenatal care coverage and health outcomes. Cochrane Database Syst Rev 2015;375.

10. Carroli G, Rooney C, Villar J. How effective is antenatal care in preventing maternal mortality and serious morbidity? An overview of the evidence. Paediatr Perinat Epidemiol 2001;15:1-42.

11. United Nations, Departement of Economic and Social Affairs, Population Division. World Population Prospects: The 2017 Revision Data Booklet (ST/ESA/SER.A/401) 2017.

12. United Nations Children's Fund. The state of the world's children: a fair chance for every child. New York, NY: UNICEF, 2016.

13. Imdad A, Bhutta ZA. Routine iron/folate supplementation during pregnancy: effect on maternal anaemia and birth outcomes. Paediatr Perinat Epidemiol 2012;26:168-77.

14. Peña-Rosas JP, De-Regil LM, Garcia-Casal MN, et al. Daily oral iron supplementation during pregnancy. Cochrane Database Syst Rev $2015 ; 170$.

15. World Health Organization. Guideline: daily iron supplementation in adult women and adolescent girls. Geneva, Switzerland World Health Organization; 2016

16. Hess SY, Ouédraogo CT, Bamba IF, et al. Using formative research to promote antenatal care attendance and iron folic acid supplementation in Zinder, Niger. Matern Child Nutr 2018;14:e12525.

17. Ridde V, Diarra A. A process evaluation of user fees abolition for pregnant women and children under five years in two districts in Niger (West Africa). BMC Health Serv Res 2009;9:89.

18. Institut National de la Statistique. Manuel des concepts et definitions. Niamey, Niger Institut National de la Statistique; 2013.

19. United Nations. Designing household survey samples: practical guidelines. New York, NY: Department of Economics and Socia Affairs, Statistics Division, United Nations, 2008.

20. Hess SY, Ouedraogo CT. NiMaNu project, 2017. Available: https:// osf.io/r68s7/ [Accessed 2 Mar 2018]. 
21. Papageorghiou AT, Ohuma EO, Gravett MG, et al. International standards for symphysis-fundal height based on serial measurements from the fetal growth longitudinal study of the INTERGROWTH-21st project: prospective cohort study in eight countries. BMJ 2016;355:i5662.

22. Begum K, Ouedraogo CT, Wessells KR, et al. Prevalence of and factors associated with antenatal care seeking and adherence to recommended iron-folic acid supplementation among pregnant women in Zinder, Niger. Matern Child Nutr 2018;14.

23. Coates J, Swindale A, Bilinsky P. Household food insecurity access scale (HFIAS) for measurement of household food access: indicator guide (v.3). Washington, DC, USA: FHI 360/FANTA, 2007.

24. FAO and FHI 360. Minimum dietary diversity for women: a guide for the measurement. Rome, Italy: FAO, 2016.

25. Guyon AB, Quinn VJ. Booklet on key essential nutrition actions messages. Washington, DC: Core Group, 2011.

26. USAID, Helen Keller International, JSI. Understanding the essential nutrition action (ENA) framework. Washington, DC: USAID, 2014.

27. Kok G, Gottlieb NH, Peters G-JY, et al. A taxonomy of behaviour change methods: an intervention mapping approach. Health Psychol Rev 2016;10:297-312.

28. Legros S, Tawfik Y, Abdallah $\mathrm{H}$, et al. Evaluation of the quality assurance project and basics joint project in Niger. Int $J$ Qual Health Care 2002;14:97-104.

29. Ververs MT, Antierens A, Sackl A, et al. Which anthropometric indicators identify a pregnant woman as acutely malnourished and predict adverse birth outcomes in the humanitarian context? PLOS Curr 2013;5.

30. Ekirapa-Kiracho E, Muhumuza Kananura R, Tetui M, et al. Effect of a participatory multisectoral maternal and newborn intervention on maternal health service utilization and newborn care practices: a quasi-experimental study in three rural Ugandan districts. Glob Health Action 2017;10:1363506.

31. Mushi D, Mpembeni R, Jahn A. Effectiveness of community based safe motherhood promoters in improving the utilization of obstetric care. The case of Mtwara rural district in Tanzania. BMC Pregnancy Childbirth 2010;10:14

32. Hajizadeh S, Tehrani FR, Simbar M, et al. Factors influencing the use of prenatal care: a systematic review. J Midwifery Reprod Health 2016;4:544-57.

33. Shivalli S, Srivastava RK, Singh GP. Trials of improved practices (tips) to enhance the dietary and iron-folate intake during pregnancy- a quasi experimental study among rural pregnant women of Varanasi, India. PLoS One 2015;10:e0137735.

34. Ojewole F, Oludipe YO. Pregnancy-related information need and information-seeking pattern among pregnant women attending antenatal clinic at Ikorodu General Hospital, Lagos state, Nigeria. Euro Sci J 2017:13:436-47.

35. Ndiaye M, Siekmans K, Haddad S, et al. Impact of a positive deviance approach to improve the effectiveness of an iron supplementation program to control nutritional anemia among rural Senegalese pregnant women. Food Nutr Bull 2009;30:128-36.

36. Garg A, Kashyap S. Effect of counseling on nutritional status during pregnancy. Indian J Pediatr 2006;73:687-92.

37. Senanayake HM, Premaratne SP, Palihawadana T, et al. Simple educational intervention will improve the efficacy of routine antenatal iron supplementation. J Obstet Gynaecol Res 2010;36:646-50.

38. Wessells K, Ouédraogo C, Young R, et al. Micronutrient status among pregnant women in Zinder, Niger and risk factors associated with deficiency. Nutrients 2017:9:430.

39. Hess SY, Ouédraogo CT, Young RR, et al. Urinary iodine concentration identifies pregnant women as iodine deficient yet school-aged children as iodine sufficient in rural Niger. Public Health Nutr 2017;20:1154-61.

40. Olney DK, Talukder A, lannotti LL, et al. Assessing impact and impact pathways of a homestead food production program on household and child nutrition in Cambodia. Food Nutr Bull 2009;30:355-69.

41. US Institute of Medicine. Weight gain during pregnancy: reexamining the guidelines. Washington, DC The National Academies Press; 2009.

42. Ota $\mathrm{E}$, Hori $\mathrm{H}$, Mori R, et al. Antenatal dietary education and supplementation to increase energy and protein intake. Cochrane Database Syst Rev 2015;41.

43. Adu-Afarwuah S, Lartey A, Okronipa $\mathrm{H}$, et al. Maternal supplementation with small-quantity lipid-based nutrient supplements compared with multiple micronutrients, but not with iron and folic acid, reduces the prevalence of low gestational weight gain in semi-urban Ghana: a randomized controlled trial. J Nutr 2017;147:697-705

44. Reynolds WM. Development of reliable and valid short forms of the Marlowe-Crowne social desirability scale. J Clin Psychol 1982;38:119-25.

45. Menon P, Nguyen PH, Saha KK, et al. Impacts on breastfeeding practices of at-scale strategies that combine intensive interpersonal counseling, mass media, and community mobilization: results of cluster-randomized program evaluations in Bangladesh and Viet Nam. PLoS Med 2016;13:e1002159.

46. Sudfeld CR, Smith ER. New evidence should inform WHO guidelines on multiple micronutrient supplementation in pregnancy. J Nutr 2019;149:359-61. 